\title{
Tuning Electrical and Optical Properties of Transparent Con- ductive Thin Films Using ITO and ZnO Nanoparticles, Sol-Gel- ZnO and Ag Nanowires
}

\author{
M Schuster", S Groß, F Roider, I Maksimenko and PJ Wellmann
}

Department of Materials Science 6, Materials for Electronics and Energy Technology, Friedrich-AlexanderUniversity Erlangen-Nürnberg, Germany

\begin{abstract}
The progress in the research on a wet-chemical or nanoparticle derived absorber layer for solar cells leads to the fabrication of a printable transparent front electrode. This eliminates the need for vacuum technology, when the vacuum chain is already breached for the absorber deposition. The objective of this work is the manufacturing and characterization of a vacuum-free, printable, transparent front electrode on the basis of conductive metal oxides like ITO and $\mathrm{ZnO}$, while reducing Indium consumption and annealing temperature or duration. Nanoparticles as well as a sol-gel process were used to deposit transparent and conductive Aluminum Doped Zinc Oxide (AZO) thin films on glass substrates. Furthermore, the influence of ion-implantation-doping after layer deposition was examined. Different temperature treatments were applied after layer deposition or ion-implantation respectively to tune the conductivity of the thin films. For characterization of the thin-films fourpoint-probe measurements, white-light-interferometry, UVIVIS spectroscopy, and (FE) SEM were used. The sol-gel-layers achieved a conductivity of $314 \mathrm{~S} / \mathrm{cm}$ whereas the nanoparticle derived $\mathrm{ZnO}$ layers only reached $1.15-20 \mathrm{~S} / \mathrm{cm}$. Finally, the application of silver nanowires resulted in a low sheet resistance of $5.8 \Omega / \square$ which is even lower than for state-of-the-art sputtered AZO-layers $(15 \Omega / \square)$ and allows low annealing temperatures of only $140{ }^{\circ} \mathrm{C}$.
\end{abstract}

\section{Keywords}

Transparent conductive film, ITO \& ZnO nanoparticles, Sol-Gel ZnO:(In,Al), Silver nanowires (AgNW)

\section{Introduction}

Transparent conductive oxides are widely used for a huge number of applications such as solar-cell window layers, electroluminescence lamps, touch screen displays and many more. The number one material used is ITO which has outstanding properties but relies on the scarce indium. Therefore, doped $\mathrm{ZnO}$ emerges more and more as an alternative to ITO. For CISe solar cells, sputtered $\mathrm{ZnO}$ window layers are commonly used. Since there is progress on solution processing of the absorber layer, the vacuum-chain is already interrupted for Chemical Bath Buffer Layer Deposition (CBD) and the scale-up of vacuum processing, necessary for large modules, is challenging, non-vacuum processes for $\mathrm{ZnO}$ deposition are interesting. Investigations have started since a long time in this field and are still performed recently [1-8], emphasizing low-temperature approaches and vacuum-free processing, or using silver nanowires or -particles for composite [9-11].

*Corresponding author: Matthias Schuster, Department of Materials Science 6, Materials for Electronics and Energy Technology, Friedrich-Alexander-University Erlangen-Nürnberg, Martensstr 7, 91058 Erlangen, Germany, Tel: +49-(0)-9131/85-27719, Fax: +49-(0)-9131/85-28495, E-mail: msc.matthias.schuster@fau.de

Received: August 01, 2017: Accepted: December 12, 2017: Published: December 14, 2017

Copyright: ( 2017 Schuster M, et al. This is an open-access article distributed under the terms of the Creative Commons Attribution License, which permits unrestricted use, distribution, and reproduction in any medium, provided the original author and source are credited. 
In this work $\mathrm{ZnO}$ and ITO Nanoparticles (NPs) were used alongside $\mathrm{ZnO}$ :Al,In sol-gel processing and Silver Nanowires (Ag-NWs) to manufacture and characterize printable, transparent, conductive thin films, with the main goals to tune optical and electrical properties to fit for various applications, as well as reduce Indium consumption and annealing temperatures. Ion-implantation doping was investigated in respect of possibility for low-temperature and Rapid Thermal Annealing (RTA), short enough that temperature sensitive substrates could handle the stress. Furthermore, nanoparticle mixtures, and infiltration-doping were investigated. Finally the potential of silver nanowires combined with a sol-gel process for $\mathrm{ZnO}$ : Al,In is shown, as conductive thin films independent on high temperature annealing were manufactured.

\section{Experimental}

\section{Sol-Gel film formation}

Aluminum nitrate hydrate $\left(\mathrm{Al}\left(\mathrm{NO}_{3}\right)_{3} \times 9 \mathrm{H}_{2} \mathrm{O}-98.8 \%\right.$ - VWR), ethanol (absolute - Merck), (indium chloride $\left(\mathrm{InCl}_{3}\right)(99.99 \%$, Alfa Aesar), isopropanol (IPA - 99.8\% - Merck), 2-methoxy ethanol (MOE - 99\% - Alfa Aesar), mono ethanol amine (MEA - $98+\%$ - Alfa Aesar), silver-nanowires (Ag-NW - Clear Ohm Silver Nanowires - Cambrios Technologies Corporation), zinc acetate hydrate $\left(\mathrm{ZnAc}_{2} \times 2 \mathrm{H}_{2} \mathrm{O}-\right.$ Merck) were used as received without further purification.

The sol synthesis followed Schuler and Aegerter [1] with minor changes. First MEA and $\mathrm{ZnAc}_{2}$ were dissolved in IPA or MOE respectively and stirred until a clear solution was acquired with concentrations of $0.1 \mathrm{M}$ and $0.5 \mathrm{M}$. Then the dopant $\mathrm{InCl}_{3}$ and/or $\mathrm{Al}\left(\mathrm{NO}_{3}\right)_{3}$ was added dissolved in ethanol. The sol then was stored in small flasks and stable to use for several weeks although we recommend using fresh sols.

For thin-film formation the sol was spin coated or doctor-bladed on float glass substrates without any seeding layer several times to achieve different film thickness and dried in between on a hotplate at $150^{\circ} \mathrm{C}$. After deposition, the xerogel film was annealed for 90 minutes in air, vacuum or $5 \% \mathrm{H}_{2}$ forming gas at temperatures from $140-550{ }^{\circ} \mathrm{C}$, mentioned in detail in results section, to form a dense film and improve conductivity. In a final step Cambrios Technologies Corporation Clear Ohm Silver Nanowires (Ag-NW) were used as received without synthesis or purification. Ag-NW were deposited on the sol-gel $\mathrm{ZnO}$ :Al,In films, annealed in vacuum at $140^{\circ} \mathrm{C}$, by doctor-blading to further reduce sheet resistance at a low annealing temperature of $140{ }^{\circ} \mathrm{C}$.

\section{Nanoparticle film formation}

Aqueous ITO and $\mathrm{ZnO}$ nanoparticle dispersions re- ceived from Evonik Degussa GmbH were deposited on float glass or fused silica by spin coating without synthesis or purification. After deposition, the layers were annealed under argon atmosphere in a tube furnace at $800{ }^{\circ} \mathrm{C}$ for 30 minutes, then ion-implanted with aluminum or infiltrated with an above-mentioned sol before being annealed again. Ion-implantation was performed using a Varian $350 D$ ion-implanter and doses of $10^{12} \mathrm{~cm}^{-2}$, $10^{13} \mathrm{~cm}^{-2}$, and $10^{14} \mathrm{~cm}^{-2}$ with up to $250 \mathrm{keV}$. This resulted in doping concentrations of $10^{16} \mathrm{~cm}^{-3}, 10^{17} \mathrm{~cm}^{-3}$ and $10^{18} \mathrm{~cm}^{-3}$. The second annealing was performed under argon atmosphere in a tube furnace or an RTA (rapid thermal annealing) furnace at $800{ }^{\circ} \mathrm{C}$ for $30 \mathrm{~min}$ and 20 secs respectively. For the infiltration process, a nanoparticle film substrate was dipped in the sol for $30 \mathrm{~s}$, so that the sol was soaked into the porous NP-film, and then dried at $60{ }^{\circ} \mathrm{C}$ for 15 minutes and annealed at $550{ }^{\circ} \mathrm{C}$ in air for 60 minutes.

\section{Characterization}

The sheet resistance of the samples was measured by four-point-probing with a Keithley 2401 Source meter to yield the conductivity, and white-light-interferometry to yield the film-thickness.

The optical properties, absorbance and transmittance, were measured from UV/VIS spectroscopy using a Perkin Elmer Lambda P950.

The porosity of the films was calculated using the Swanepoel-Method. Firstly, the refractive index of the substrate was calculated from maximum transmission. Then maximum and minimum transmission for the thin film were approximated by the enveloping functions of the interference pattern and again used to calculate the refractive index. Finally, the porosity $X$ was calculated from the respective refractive indices $n$ of air, bulk and the film:

$$
X=\frac{n_{\text {film }}-n_{\text {bulk }}}{n_{\text {air }}-n_{\text {bulk }}}
$$

The morphology was investigated on a Jeol 6400 JSM scanning electron microscope.

XRD spectra were conducted on a PANalytical Empyrean Diffractometer with $\mathrm{Cu}-\mathrm{Ka}$ radiation and Bragg-Brentano set-up.

\section{Results and Discussion}

To understand the effects of ion implantation and annealing on the commercially received $\mathrm{ZnO}$ nanoparticles, at first the as deposited $\mathrm{ZnO}$ films were investigated. Figure 1 shows $\mathrm{ZnO}$ films directly after deposition and after the first annealing at $800^{\circ} \mathrm{C}$. It can be seen that prior to the annealing particles of different sizes lie next to each other and are not connected. During the annealing, 

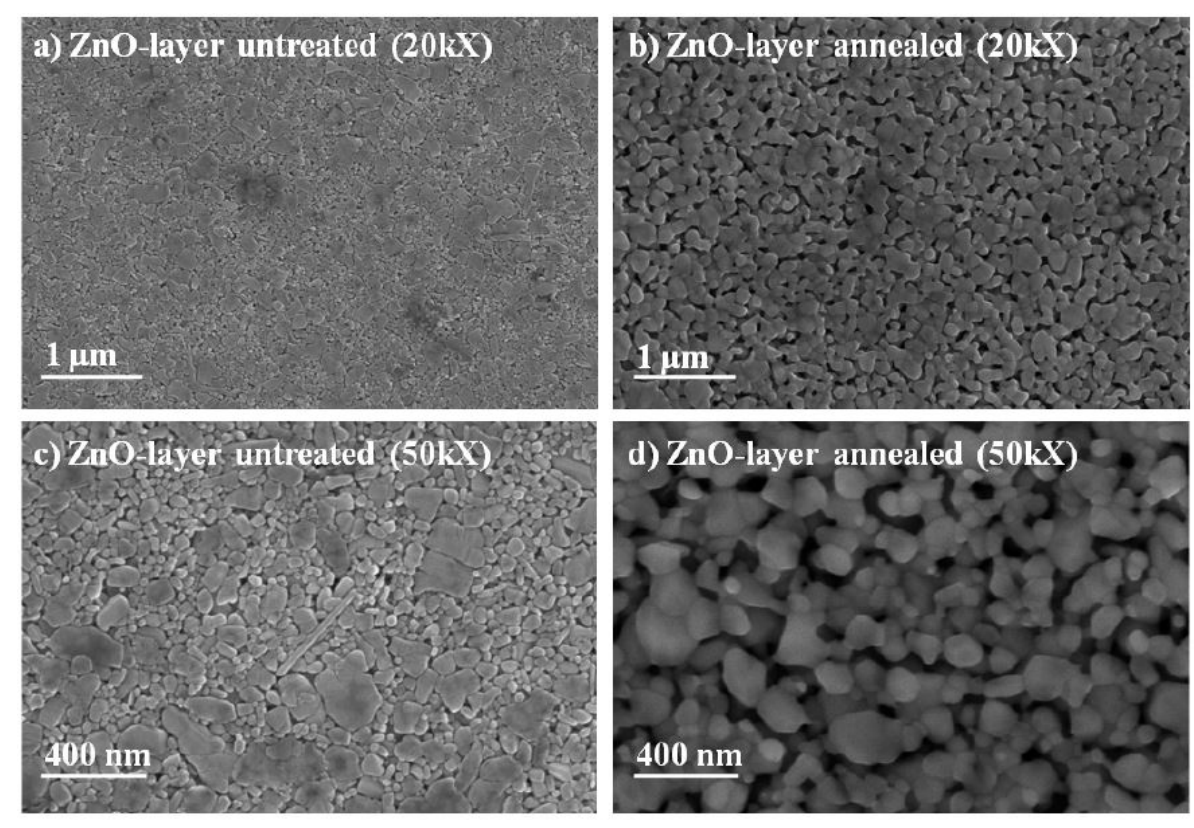

Figure 1: SEM top view of as deposited and annealed $\mathrm{ZnO}$ nanoparticle films. The annealing at $800{ }^{\circ} \mathrm{C}$ sinters the particles together, while also reducing the layer thickness from $630 \mathrm{~nm}$ to $560 \mathrm{~nm}$.

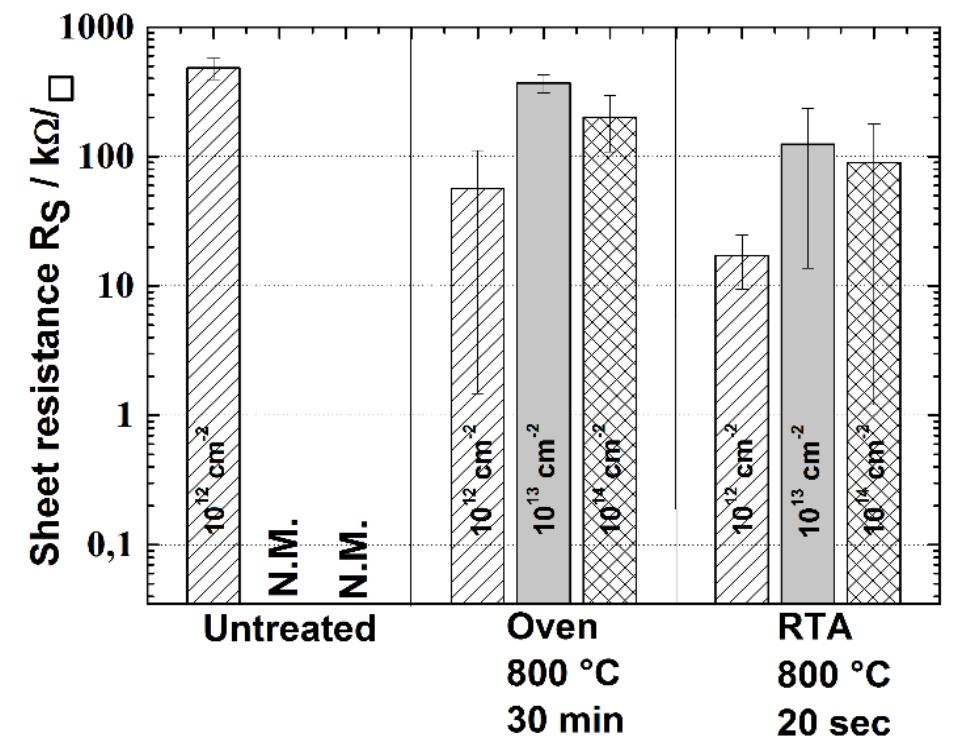

Figure 2: Sheet resistance for different post-ion-implantation-annealing. Untreated samples of $10^{13}$ and $10^{14} \mathrm{~cm}^{-2}$ were not measurable. Tube furnace annealing and Rapid Thermal Annealing (RTA) reduce sheet resistance significantly. The best result is achieved with $10^{12} \mathrm{~cm}^{-2}$ dose and RTA.

the particles sinter together consuming smaller particles, minimizing the surface energy. Thus, the number of grains and grain boundaries is decreased. Moreover, many sintering necks form, connecting the particles, so in total the porosity decreases from $47 \%$ to $40 \%$. Also, the layer thickness changes ca. $10 \%$ from $626 \pm 31 \mathrm{~nm}$ before to $564 \pm 33 \mathrm{~nm}$ after the first heat treatment. Now the effects of ion-implantation doping of $\mathrm{ZnO}$ nanoparticle films were studied. Figure 2 shows the sheet resistance of the different sample series processed with different post-implantation annealing. It is notable, that for doses of $10^{13} \mathrm{~cm}^{-2}$ and $10^{14} \mathrm{~cm}^{-2}$ the four-point-probe measurement did not deliver any result right after implantation. This shows the huge influence of radiation damage in the crystallites, diminishing the conductivity to minimum values. The heat treatment at $800{ }^{\circ} \mathrm{C}$ heals the radiation damage and activates the dopant, thus enhancing the conductivity and reducing the sheet resistance while the layer thickness is not reduced any further. Interestingly in both annealing processes, the samples implanted with $10^{12} \mathrm{~cm}^{-2}$ show the lowest resistance, despite having the lowest doping concentration. This could as well be ex- 

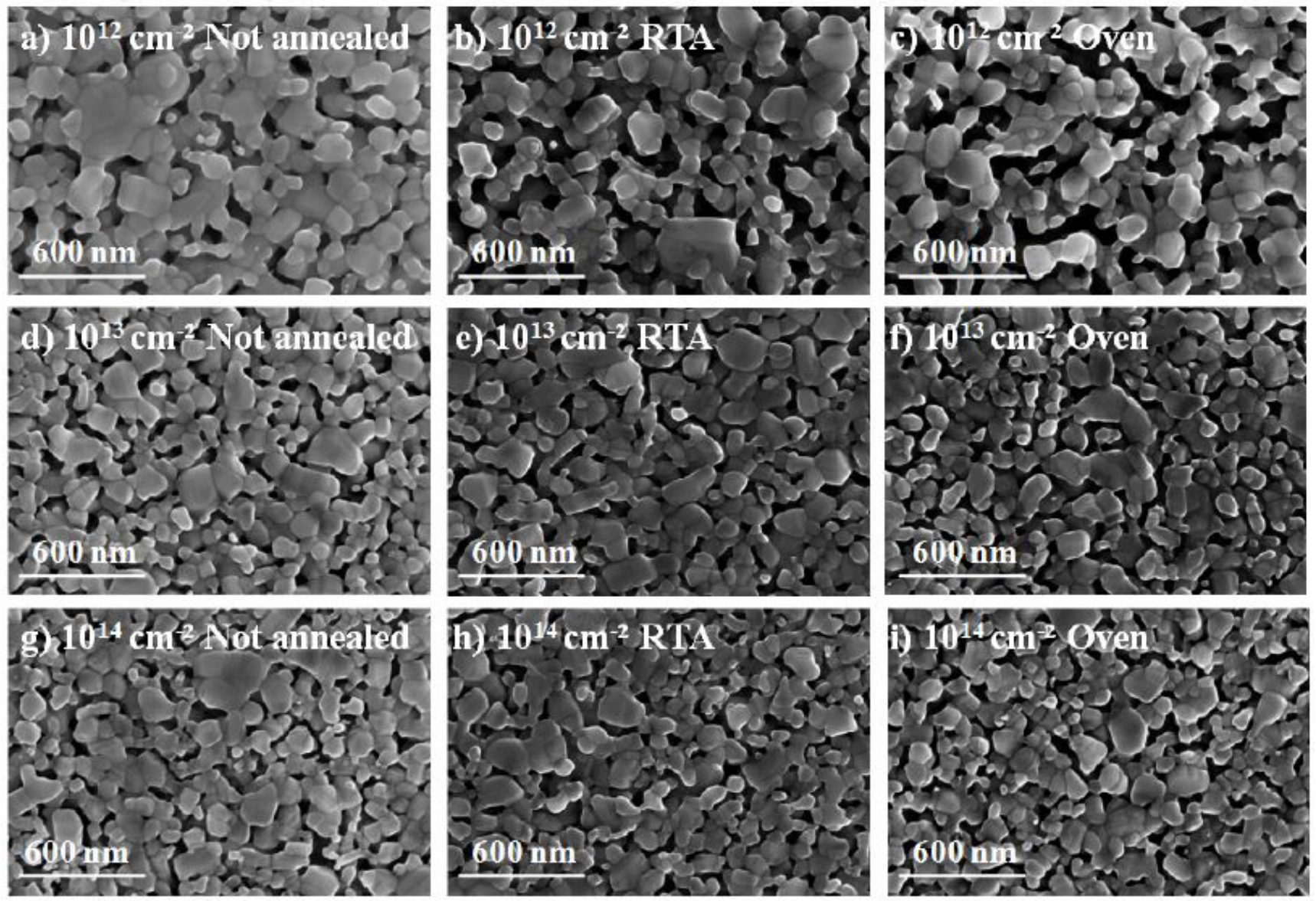

Figure 3: SEM top view of ion implanted ZnO nanoparticle films after implantation with different doses, after RTA and after annealing in furnace. Higher doses radiation damage breaks sintering necks between grains which is only slightly repaired by annealing.

plained with greater radiation damage from larger implantation doses that might be too severe to be healed during annealing. Using larger doses than $10^{13} \mathrm{~cm}^{-2}$ again benefits the conductivity by having more dopants, enhancing the conductivity more than the radiation damage diminishes it. Furthermore, it can be seen, that the RTA samples have smaller sheet resistance than from the longer annealing in the tube furnace. This results from the very short annealing times, activating the dopants, but not allowing thermal diffusion. This keeps the doping profile as desired and does not broaden it, resulting in higher conductivity. The morphology of nanoparticle films, as depicted in Figure 3, shows porous layers with sinter necks from the first heat treatment, but the implantation seems to have destroyed some sinter necks. The second heat treatment did not improve the surface morphology by much, neither the RTA nor if held for longer times, as the particles did not grow further or form new sinter necks. It can be seen, that for the smallest dose of $10^{12} \mathrm{~cm}^{-2}$, the grains are the largest and the porosity is ca. $42 \%$. Greater doses result in greater destruction of sinter necks and introduce more grain boundaries such leaving fewer pathways for the electrons. This explains the larger sheet resistance for the higher doses very well. In the case of $10^{13} \mathrm{~cm}^{-2}$ and $10^{14} \mathrm{~cm}^{-2}$ the porosity is comparable with $36 \%$ and $35 \%$ respectively, and notably smaller than for $10^{12} \mathrm{~cm}^{-2}$. It seems the ion implantation can lead to densification, possibly by breaking particles apart that can then fill the pores better. Also, the expected larger doping concentration from the larger implantation dose again lowers the sheet resistance from $10^{13} \mathrm{~cm}^{-2}$ to $10^{14} \mathrm{~cm}^{-2}$. The best result for conductivity was $\sigma=1.15 \mathrm{~S} \mathrm{~cm}^{-1}$ for dose $10^{12} \mathrm{~cm}^{-2}$ and RTA, even though the porosity is a bit larger and the doping concentration lower compared to the higher doses. This shows the importance of the sinter necks and having fewer grain boundaries, as well as a very short RTA process, so that temperature sensitive substrates could handle the stress. However, the results need more improvement for any application as transparent electrode.

Infiltration of a $\mathrm{ZnO}$ nanoparticle film with a 4.5 at $\%$ in and 0.5 at $\% \mathrm{Al}$ doped $\mathrm{ZnO}$-sol resulted in an enhancement of conductivity from $2 \times 10^{-3}$ to $8 \times 10^{-2} \mathrm{~S} \mathrm{~cm}^{-1}$. However, the increase did not reach the values achieved with ion-implantation as only the sol was doped and the $\mathrm{ZnO}$ NPs themselves only received minor doping from 

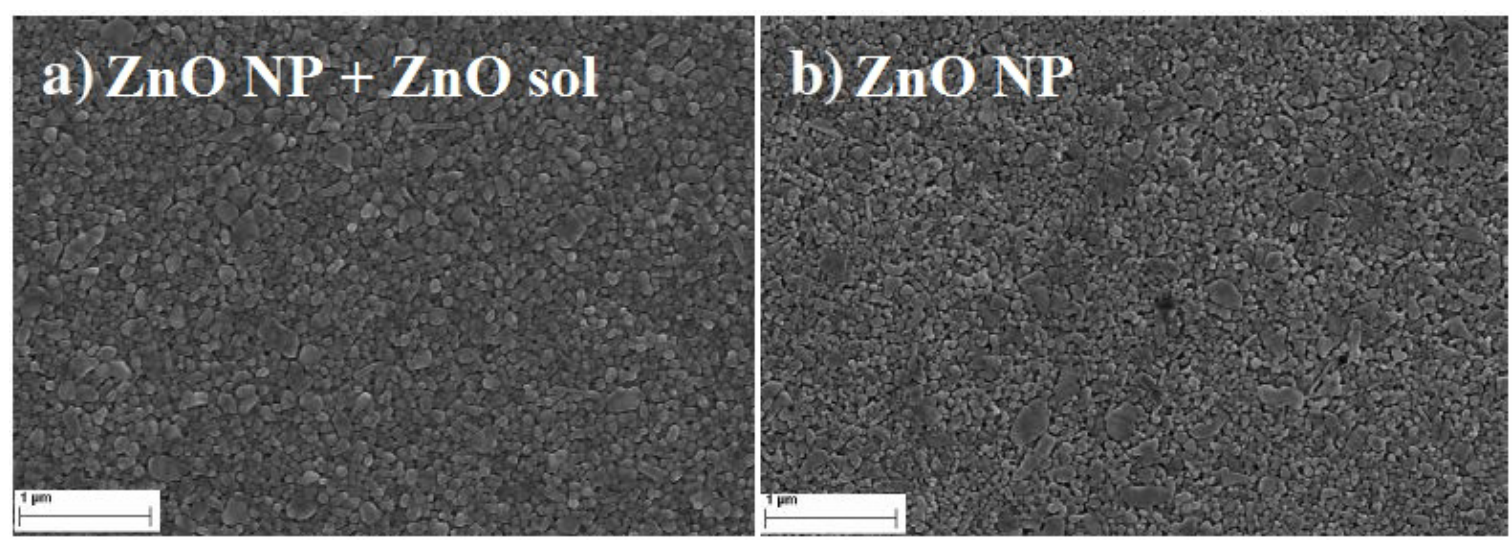

Figure 4: SEM top view of $\mathrm{ZnO}$ nanoparticle films before and after infiltration with doped ZnO-sol. Wetting of ZnO particles with the sol enhances contact area and sintering neck formation as well as filling the empty spaces between particles.

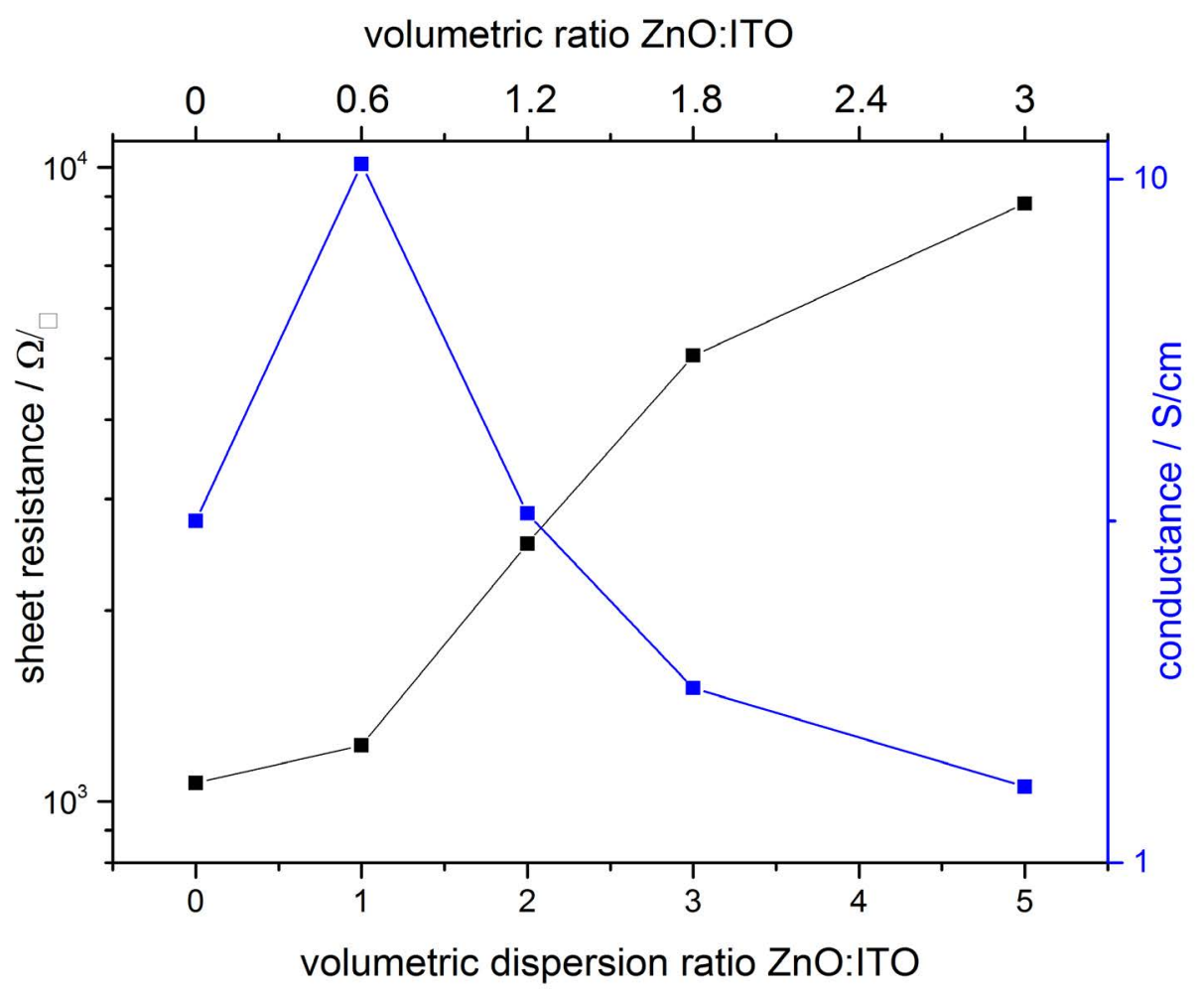

Figure 5: Sheet resistance and conductance for different mixtures of $\mathrm{ZnO}$ and ITO nanoparticles. The best result is achieved with a volumetric ratio of $\mathrm{ZnO}: \mathrm{ITO}$ of 0.6 .

the sol by thermal diffusion during annealing process. This sol alone, without ZnO NPs, achieves a conductivity of $0.1 \mathrm{~S} \mathrm{~cm}^{-1}$ in a $100 \mathrm{~nm}$ film. Interestingly, lower doped sols achieved a larger conductivity as a film without $\mathrm{ZnO}$ NPs but performed worse than the higher doped sol when trying to enhance the $\mathrm{ZnO}$ NP layer. Film thickness was changed ca. $20 \mathrm{~nm}$ by the infiltration process, being within standard deviation of pure ZnO NPs films. Transmission behavior did not change significantly. The conductive pathways most likely consist of the doped matrix from the sol and the surface of $\mathrm{ZnO} N P$. The morphology was improved a little bit, as shown in Figure 4, as the spaces between the particles are filled but the pores could not be closed by infiltration completely. The particles achieved a rounder shape and grew to a diameter of $72 \pm 23 \mathrm{~nm}$ from previously $62 \pm 25 \mathrm{~nm}$ as well as more sintering necks were formed. This is due to the wetting of particles with the sol, making the contact area larger and thus enhancing the sintering process. Still the electrical properties need more improvement for application.

To improve the conductivity with wet-chemical processes, mixtures of $\mathrm{ZnO}$ and ITO nanoparticles were transformed into films and characterized. In Figure 5 conductance and sheet resistance for different mixtures is shown. Adding more and more $\mathrm{ZnO}$ particles enhanc- 


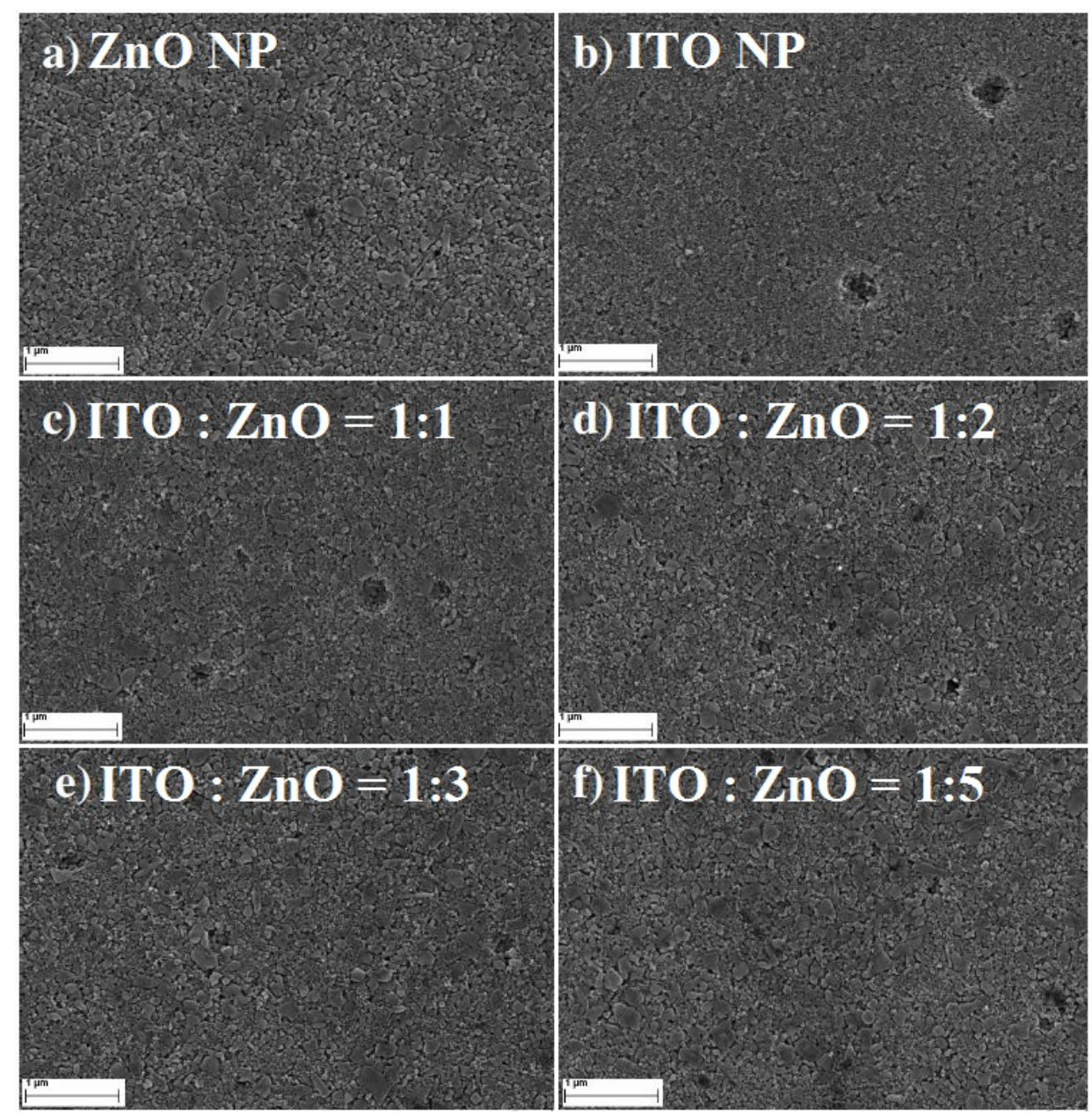

Figure 6: SEM top view of different mixtures of $\mathrm{ZnO}$ and ITO nanoparticles shows good intermixing with no tendencies of separation. The higher the $\mathrm{ZnO}$ amount the more the morphology resembles the pure $\mathrm{ZnO}$ morphology with broader particle size distribution than pure ITO. Moreover, mixed films seem more densely packed than pure $\mathrm{ZnO}$ films.

es the sheet resistance, as the undoped $\mathrm{ZnO}$ particles possess a lower conductivity than the ITO particles and less conductive pathways between ITO NPs remain. However also the film thickness is reduced from $1.8 \mu \mathrm{m}$ to $0.8 \mu \mathrm{m}$, so in conclusion the conductivity rises to $10.2 \mathrm{~S}$ $\mathrm{cm}^{-1}$ at a volumetric ratio of $\mathrm{ZnO}$ :ITO $=0.6$ (= dispersion ratio 1:1). After this, the lower conductivity of $\mathrm{ZnO}$ and decreasing number of ITO paths dominates over the further reduction of layer thickness to $0.5 \mu \mathrm{m}$ and the overall conductivity drops again. The sheet resistance values for pure ITO and the $0.6 \mathrm{ZnO}$-ITO mixtures are quite similar with ca. $1.0 \mathrm{k} \Omega / \square$ and ca. $1.3 \mathrm{k} \Omega / \square$ respectively. This opens the possibility to save scarce Indium by adding $\mathrm{ZnO}$. The changes in morphology are shown in Figure 6. It can clearly be seen that the ITO NPs are smaller than the $\mathrm{ZnO}$ NPs and have a narrower size distribution, although exact values have not been measured. Moreover, ITO NP films show craters from evaporating solvents. All mixtures show intermixed particles with no preferred agglomeration and the expected rising number of larger $\mathrm{ZnO}$ particles. The 1:1 mixture, that possesses the best conductivity, shows still some craters like the pure ITO particles, which disappear for larger $\mathrm{ZnO}$ ratios. Moreover, the mixed films seem to be more densely packed than the pure films, leading to more contact area between particles and thus enhancing conductivity. However, adding more $\mathrm{ZnO}$ lowers conductivity as the larger contact area is outweighed by the low conductivity of $\mathrm{ZnO}$ particles and less contacts in-between ITO particles. The transmittance of all produced films is larger than $80 \%$ in the visible region as can be seen in Figure 7 , when reflected and diffracted light is included by measuring with an Ulbricht-sphere. The spectra of all mixtures strongly resemble the spectra of pure $\mathrm{ZnO}$, regardless of mixture ratio, and show lower transmittance than pure ITO. This might be due to the larger $\mathrm{ZnO}$ particles, which have a size close to or in the visible region of light thus scattering in all directions. This might be interesting for lighting application where light extraction in all directions is desired. The enhancement in conductivity of $\mathrm{ZnO} \mathrm{NP}$ films adding ITO nanoparticles is better by a large amount compared to the infiltration of $\mathrm{ZnO} \mathrm{NP}$ 


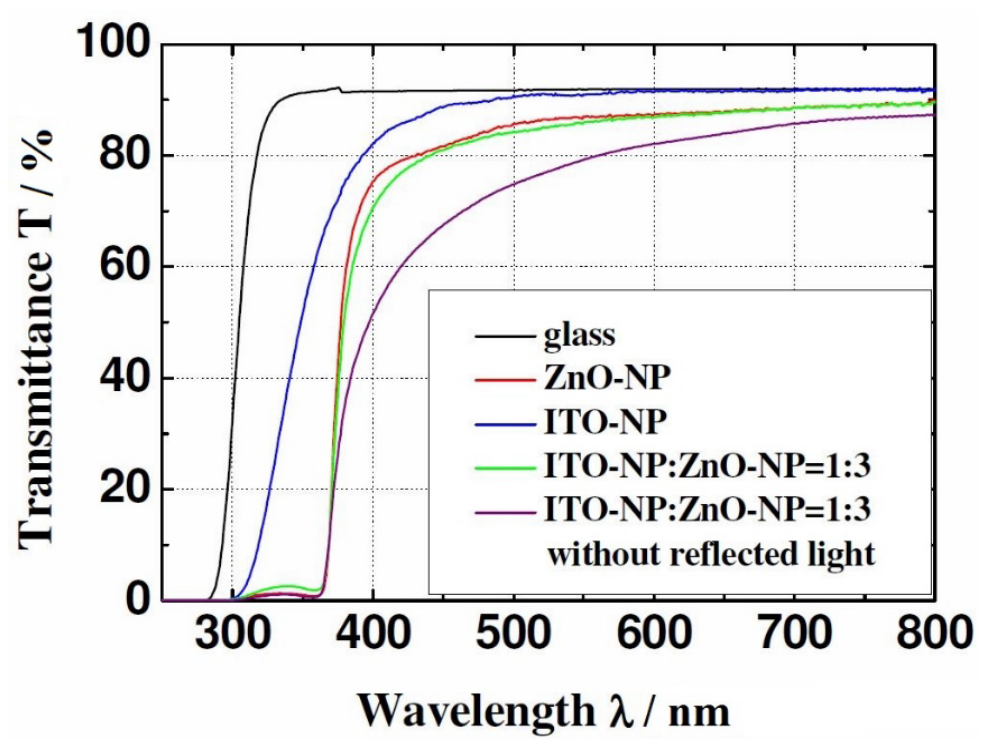

Figure 7: Wavelength dependent change in transmittance for pure $\mathrm{ZnO}$, ITO as well as a mixture. All mixtures resemble the behavior of pure $\mathrm{ZnO}$ films independently of mixing ratio, thus only one exemplary spectrum is shown.

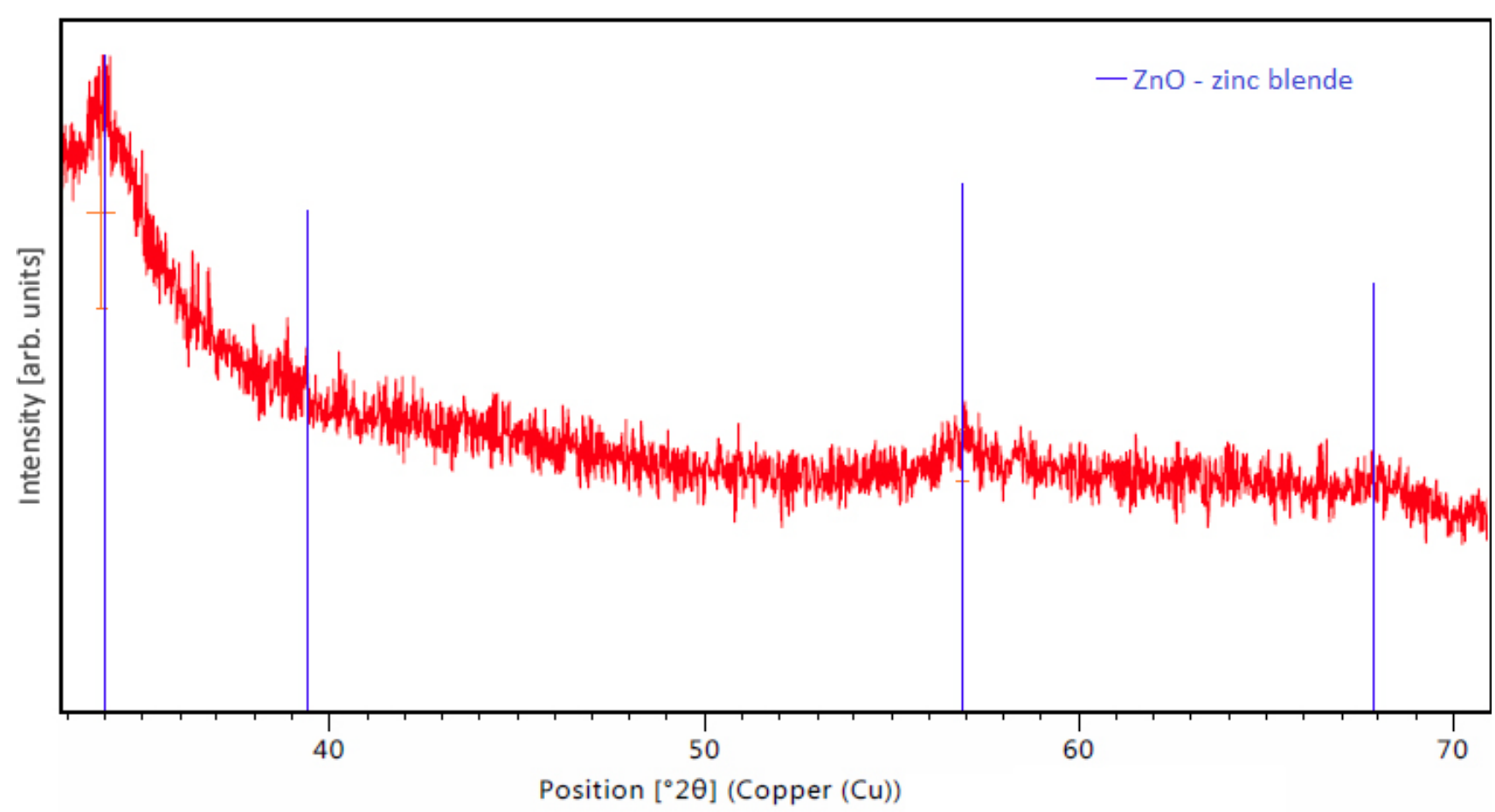

Figure 8: XRD spectrum of an as-deposited sol-gel $\mathrm{ZnO}$ thin film after drying at $230^{\circ} \mathrm{C}$. Zinc blende structure $\mathrm{ZnO}$ can be identified and no by-products are present.

films with the doped $\mathrm{ZnO}$-sol. These values are a little bit too high for application as touch screen materials, mainly due to the porosity, but there may be further room for improvement that allows application for ITO- $\mathrm{ZnO}$ nanoparticle electrodes, that reduce the Indium consumption.

As the nanoparticle films all were rather porous, a solgel method was used to form more dense $\mathrm{ZnO}$ :Al, In films. In Figure 8 an XRD spectrum of an as-deposited $\mathrm{ZnO}$ thin film can be seen, showing the reference for the identified $\mathrm{ZnO}$ zinc blende structure as well. The first step for improvement of conductivity was to find the ideal doping concentrations and the second step investigated the annealing parameters. The film thickness for all investigated films was on average $96 \pm 6 \mathrm{~nm}$. Figure 9 shows the sheet resistance for different doping concentrations where the red circle represents a purely In-doped sol, the blue diamond stands for a sol fixed at 0.5 at $\%$ In and vari- 


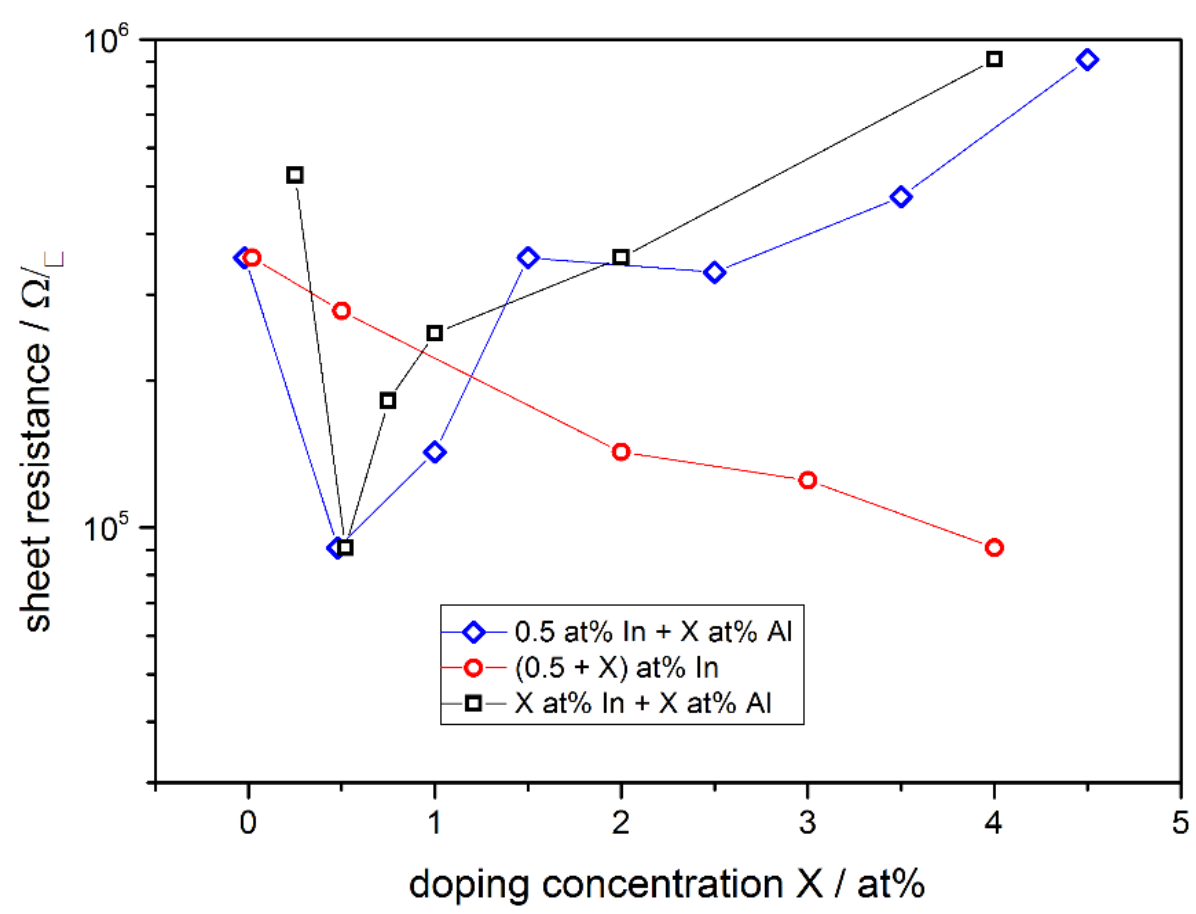

Figure 9: Sheet resistance of $\mathrm{ZnO}$ sol-gel films for different doping concentrations. The red circle shows a purely In-doped sol, the blue diamond represents a sol fixed at 0.5 at $\%$ In and variation of Al content, and the black square stands for an equal doping of In and Al.

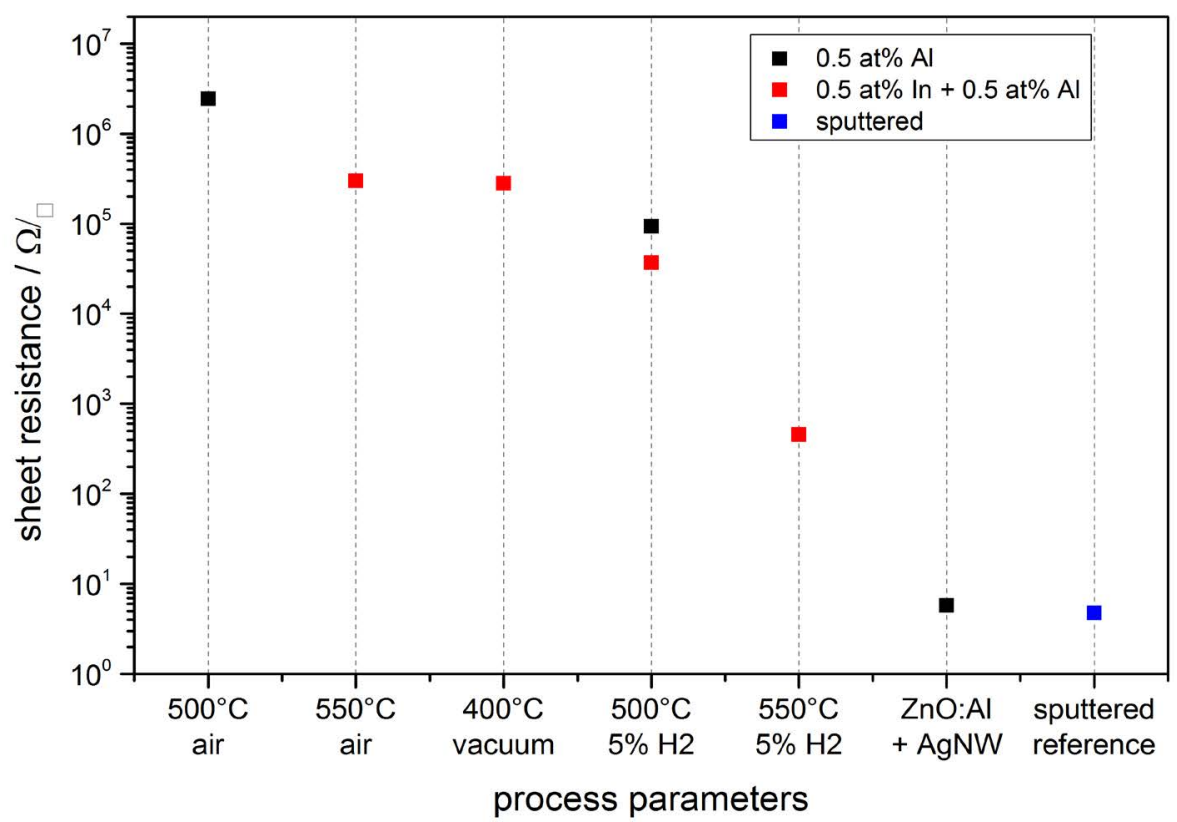

Figure 10: Sheet resistance of $\mathrm{ZnO}$ sol-gel films with different annealing parameters. The temperature was varied from 400$550{ }^{\circ} \mathrm{C}$ and the atmosphere changed from air to vacuum to $5 \% \mathrm{H}_{2}$ in $\mathrm{N}_{2}$ forming gas. Finally, the sample with Ag-NW and a $1 \mu \mathrm{m}$ sputtered reference are added to the figure as a comparison.

ation of $\mathrm{Al}$ content and the black square shows an equal doping of In and Al. With the amount of In rising the sheet resistance decreases, as well as it increases for rising $\mathrm{Al}$ amount. The more $\mathrm{Al}$ is added to the sol, it is likely to form non-conductive $\mathrm{ZnAl}_{2} \mathrm{O}_{4}$ compounds that diminish the overall conductivity. The homogeneously doped sample series shows that the effects of Al dominate over the benefits of In. The optimal doping concentration is given by the sheet resistance minimum for $0.5 \mathrm{at} \%$ In and Al. Figure 10 shows the sheet resistance for the different annealing parameters of the sol-gel $\mathrm{ZnO}$ film. It can be seen, that the atmosphere has a critical influence on the sheet resistance. Using vacuum over air yields a similar value at $400{ }^{\circ} \mathrm{C}$ compared to $550{ }^{\circ} \mathrm{C}$. This is attributed to 

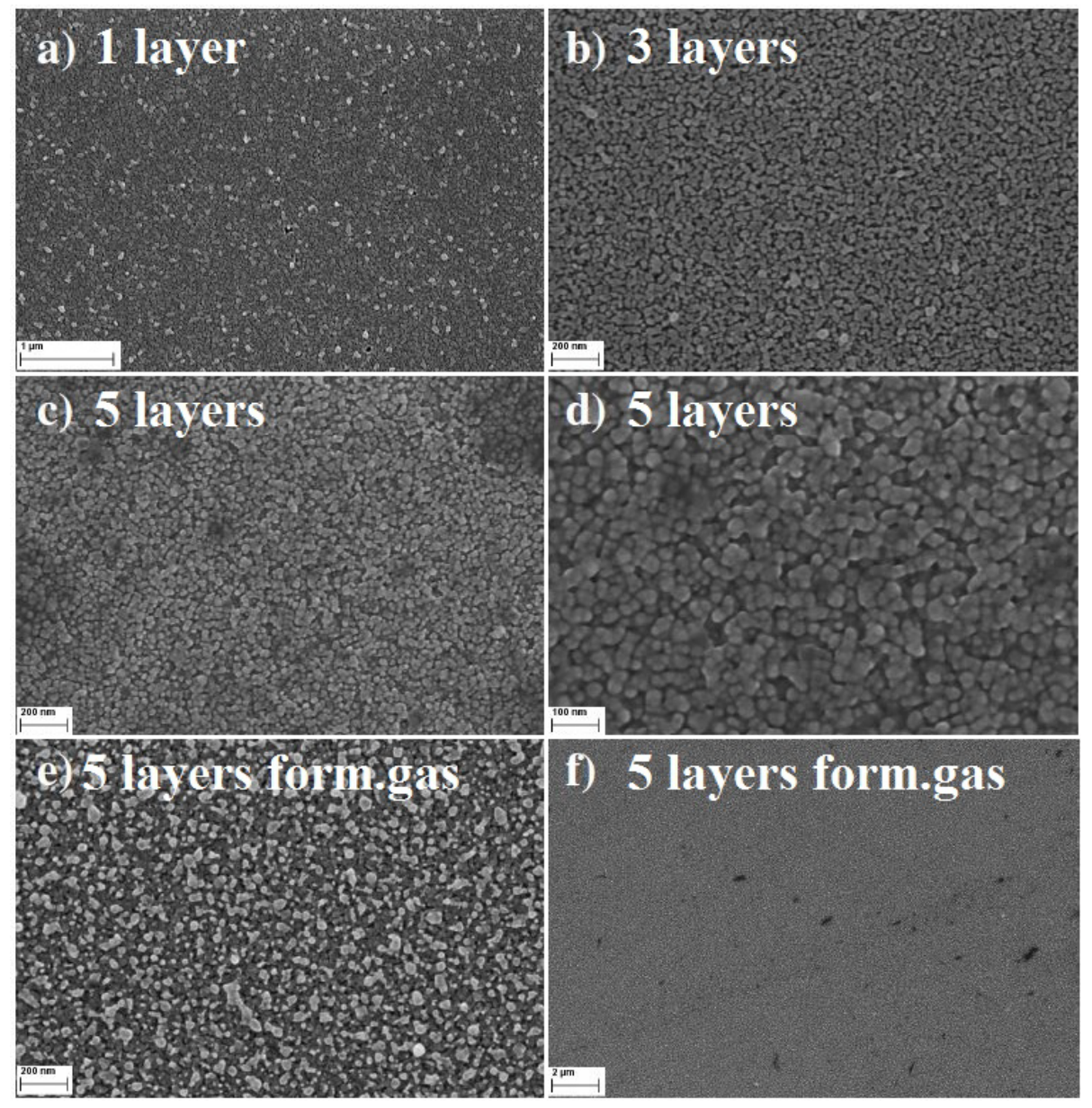

Figure 11: SEM top view of $\mathrm{ZnO}$ films with multiple coatings annealed in air or $5 \% \mathrm{H}_{2}$ in $\mathrm{N}_{2}$ forming gas at $550{ }^{\circ} \mathrm{C}$. Repeated sol coating infiltrates previously formed crystals, enhancing sintering behavior and lowering porosity. The atmosphere does not seem to have huge influence on morphology.

the enhanced formation of oxygen vacancies $\mathrm{V}_{\mathrm{O}}$ which contribute to the conduction. Annealing in forming gas $\left(5 \% \mathrm{H}_{2}\right.$ in $\mathrm{N}_{2}$ ) introduces interstitial $\mathrm{H}$ Atoms $\mathrm{H}_{\mathrm{i}}$ additionally to the $\mathrm{V}_{\mathrm{O}}$ that decrease the sheet resistance by a few orders of magnitude compared to the annealing in air. A resistance of $455 \Omega / \square$ was achieved with a conductivity of $314 \mathrm{~S} \mathrm{~cm}^{-1}$. These values are a great enhancement compared to all the nanoparticle systems but still are greatly larger than the sputtered reference, most likely due to lower crystallinity and fewer percolation paths of the sol-gel films compared to the really dense sputtered films. The porosity in sol-gel films, although smaller than for the nanoparticle films, also allows the in diffusion of oxygen, filling the $\mathrm{V}_{\mathrm{O}}$ and decreasing conductivity faster than for dense films. The morphology of sol-gel-films of different thickness annealed in air and after forming gas annealing is shown in Figure 11. At the highest magnification (d) The sinter necks between the crystallites can clearly be seen. There are no huge changes visible and the films themselves look homogeneous. By coating several times, the sol infiltrates the gaps between the firstly formed crystallites and closes the gaps during annealing, resulting in denser films as compared to the nanoparticle films. In Figure 12 an overview is given over the average transmittance of the sol-gel derived thin-films. The optical properties of all the different films were investigated with UV/VIS spectroscopy. All films show a transmittance greater than $87 \%$ which would allow them to be used for solar-cell electrodes with ease. For the samples treated with forming gas, the influence of $\mathrm{V}_{\mathrm{O}}$ and $\mathrm{H}_{\mathrm{i}}$ can be seen as a drop-in transmittance as these defects absorb some of the light below the band-gap energy. This $\mathrm{ZnO}$ :Al,In film could work as an electrode for electro-luminescence lamps or for touch screen applications as it fulfills the sheet resistance requirements. However, for an application in CISe Solar Cells the required annealing temperature is too large, as p-n-junction and CdS buffer layer will be destroyed at temperatures higher than $200^{\circ} \mathrm{C}$. By depositing Silver Nanowires (Ag-NW) on top of a sol-gel $\mathrm{ZnO}$ :Al, In film, annealed at $150^{\circ} \mathrm{C}$ in vacuum, the resulting composite can be used for solar cells. The sheet resistance reaches $5.8 \Omega / \square$ which is lower than AgNW on glass $(7 \Omega / \square)$ and state-of-the-art $400 \mathrm{~nm}$ sputtered AZO-layers $(15 \Omega / \square)$ [12]. The samples with Ag- 


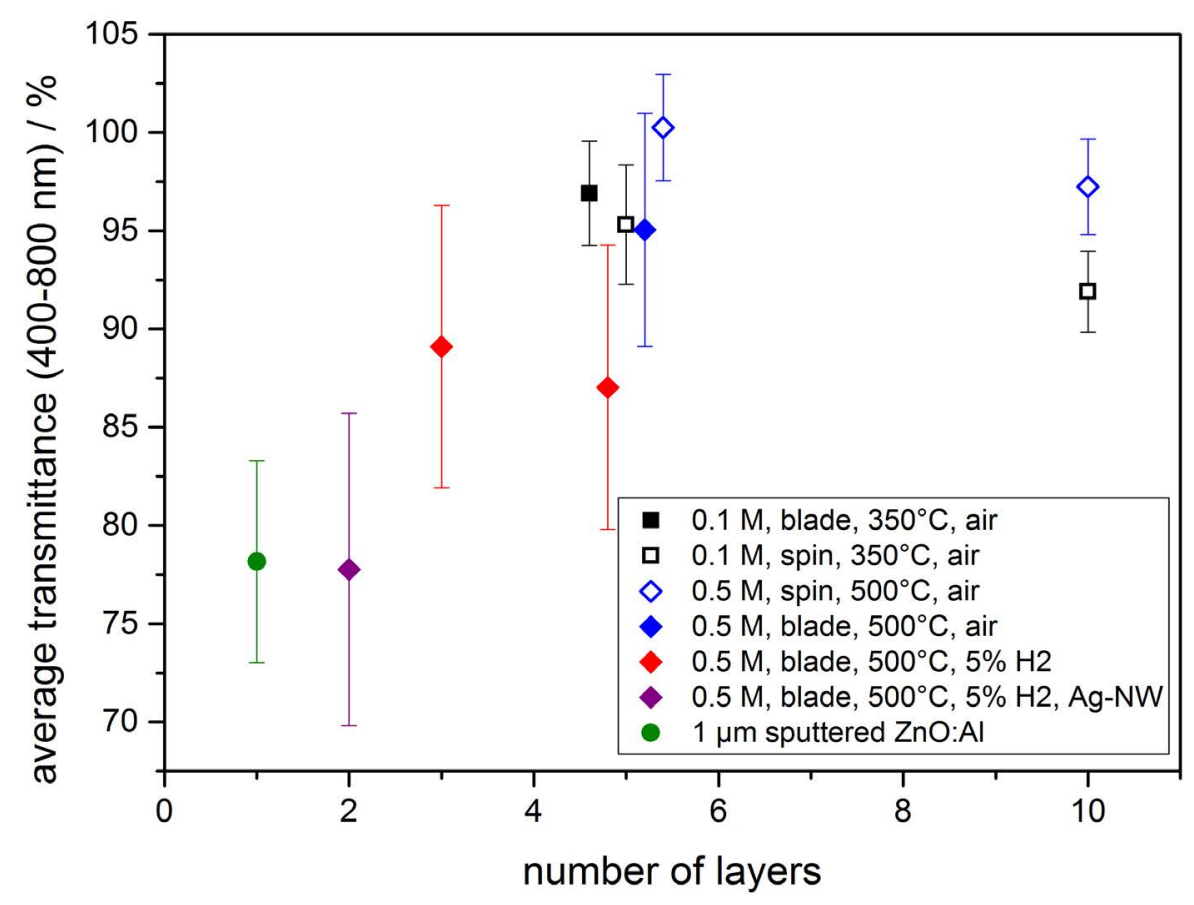

Figure 12: Average transmittance of different sol-gel derived $\mathrm{ZnO}: \mathrm{Al}, \mathrm{In}$ films. Deposition methods were spin coating and doctor-blading, the annealing was performed in air and under $5 \% \mathrm{H}_{2}$ in $\mathrm{N}_{2}$ forming gas. Finally, the sample with Ag-NW is shown as well as a $1 \mu \mathrm{m}$ sputtered reference as a comparison.

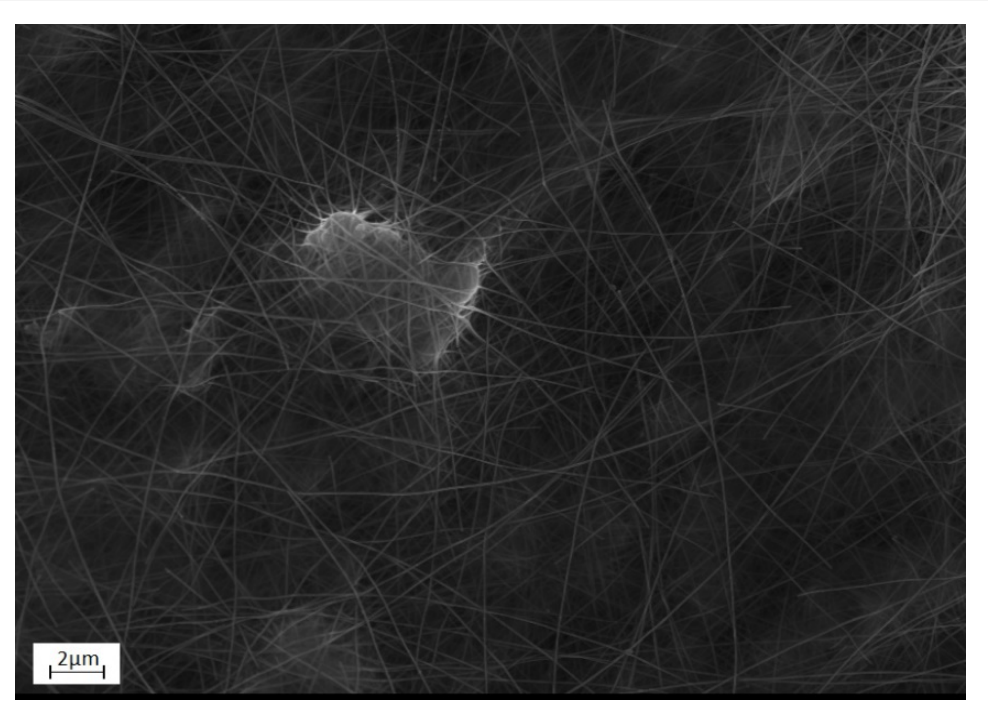

Figure 13: Percolation network of silver nanowires (Ag-NW) on top of sol-gel derived ZnO:Al film.

NW show an average transmission value of $78 \%$, which is not too far-off of the required $80 \%$ for solar-cells, at an annealing temperature as low as $140{ }^{\circ} \mathrm{C}$. The current uses the percolation network of the nanowires, seen in Figure 13, allowing thinner $\mathrm{ZnO}$ matrix films which do not need to have a very low sheet resistance themselves, thus allowing low-temperature $\mathrm{ZnO}$ for CISe solar cells.

\section{Conclusions}

In this work, different processes were used to form a transparent conductive layer and tune the electrical and optical properties. The transmittance was larger than $80 \%$ in almost all cases. Ion-implantation doping of $\mathrm{ZnO}$ nanoparticles led to a conductivity of $1.15 \mathrm{~S} \mathrm{~cm}^{-1}$ with $564 \pm 33 \mathrm{~nm}$ thickness after Rapid Thermal Annealing (RTA) treatment at $800{ }^{\circ} \mathrm{C}$ for $20 \mathrm{sec}$. Infiltrating $\mathrm{ZnO}$ nanoparticle films with doped $\mathrm{ZnO}: \mathrm{Al}$,In sols only resulted in $8 \times 10^{-2} \mathrm{~S} \mathrm{~cm}^{-1}$. Films from a mixture of $\mathrm{ZnO}$ and ITO nanoparticles with a volumetric ratio ZnO:ITO of 0.6 achieved a conductivity of $10.2 \mathrm{~S} \mathrm{~cm}^{-1}$ with a thickness of $780 \mathrm{~nm}$ and a sheet resistance of ca. $1.3 \mathrm{k} \Omega / \square$ which opens a possibility of reduction of In- 
dium consumption and practical use for touch-screens if the parameters are further optimized. Moreover solgel $\mathrm{ZnO}: \mathrm{Al}$,In films were prepared and reached a conductivity of $314 \mathrm{~S} \mathrm{~cm}^{-1}$ with a thickness of $70 \mathrm{~nm}$ and a sheet resistance of $455 \Omega / \square$ which could work for touchscreens and electro-luminescence lamps. By applying silver nanowires, the sheet resistance drops to $5.8 \Omega / \square$ at a low annealing temperature of only $140^{\circ} \mathrm{C}$, allowing usage for solar-cell electrodes and temperature sensitive substrates.

\section{Acknowledgements}

The authors would like to thank Deutsche Forschungsgemeinschaft under contract GRK 1161 and Energy Campus Nürnberg for financial support and the cluster of excellence EAM.

\section{References}

1. T Schuler, MA Aegerter (1999) Optical, electrical and structural properties of sol gel ZnO:Al coatings. Thin Solid Films 351: 125-131.

2. T Minami (2005) Transparent conducting oxide semiconductors for transparent electrodes. Semiconductor Science and Technology 20: 35-44.

3. H Oh, J Krantz, I Litzov, T Stubhan, L Pinna, et al. (2011) Comparison of various sol-gel derived metal oxide layers for inverted organic solar cells. Solar Energy Materials and Solar Cells 95: 2194-2199.

4. M Miyake, H Fukui, T Hirato (2012) Preparation of Al-doped ZnO films by aqueous solution process using a continuous circulation reactor. Physica Status Solidi 209: 945-948.
5. H Hagendorfer, K Lienau, S Nishiwaki, CM Fella, L Kranz, et al. (2014) Highly transparent and conductive ZnO: Al thin films from a low temperature aqueous solution approach. Adv Mater 26: 632-636.

6. P Fuchs, H Hagendorfer, YE Romanyuk, AN Tiwari (2015) Doping strategies for highly conductive Al-doped ZnO films grown from aqueous solution. Physica Status Solidi 212: 51-55.

7. T Kuwabara, X Wang, T Kusumi, T Yamaguchi, T Taima, et al. (2016) Flexible inverted polymer solar cells fabricated in air at low temperatures aqueous solution. Japanese Journal of Applied Physics 55.

8. YQ Liu, DD Zhang, HX Wei, QD Ou, YQ Li, et al. (2017) Highly efficient quantum-dot light emitting diodes with solgel $\mathrm{ZnO}$ electron contact. Optical Materials Express 7: 2161-2167.

9. J Krantz, M Richter, S Spallek, E Spiecker, CJ Brabec (2011) Solution-Processed Metallic Nanowire Electrodes as Indium Tin Oxide Replacement for Thin-Film Solar Cells. Advanced Functional Materials 21: 4784-4787.

10. T Stubhan, J Krantz, N Li, F Guo, I Litzov, et al. (2012) High fill factor polymer solar cells comprising a transparent, low temperature solution processed doped metal oxide/metal nanowire composite electrode. Solar Energy Materials and Solar Cells 107: 248-251.

11. B Wei, S Pan, T Wang, Z Tian, G Chen (2016) Solution-processed Ag-nanowire/ZnO- nanoparticle composite transparent electrode for flexible organic solar cells. Nanotechnology 27: 505208.

12. ZZ You, GJ Hua, YC Yong, H Jin (2012) Microstructural and optoelectrical properties of transparent conducting ZnO:Al thin films for organic solar cells. Crystal Research \& Technology 47: 1039-1046. 